\title{
Soluble VE-cadherin in metastatic breast cancer: an independent prognostic factor for both progression-free survival and overall survival
}

Pauline Rochefort ${ }^{1}$, Sylvie Chabaud ${ }^{2}$, Jean-Yves Pierga ${ }^{3}$, Olivier Tredan ${ }^{1}$, Etienne Brain ${ }^{4}$, François-Clément Bidard ${ }^{3}$, Camille Schiffler ${ }^{2}$, Helena Polena ${ }^{5,6,7}$, Abir Khalil-Mgharbel ${ }^{5,6,7}$, Isabelle Vilgrain ${ }^{5,6,7}$ and Thomas Bachelot ${ }^{\star, 1}$

${ }^{1}$ Department of Medical Oncology, Centre Léon Bérard, 28 Rue Laennec, Lyon F-69373, France; ${ }^{2}$ Direction de la Recherche Clinique et de I'Innovation, Centre Léon Bérard, Lyon F-69373, France; ${ }^{3}$ Department of Medical Oncology, Institut Curie, 26 Rue d'Ulm, Paris F-95005, France; ${ }^{4}$ Department of Medical Oncology, Institut Curie - Hopital René Hugueneau, 35 Rue Dailly, Saint Cloud F-92210, France; ${ }^{5}$ INSERM, UMR-S 1036, Grenoble F-38054, France; ${ }^{6}$ University Grenoble-Alpes, Grenoble F-38000, France and ${ }^{7} C E A, D R F$, Biosciences and Biotechnology Institute of Grenoble (BIG), Laboratory of Biology of Cancer and Infection, Grenoble F-38054, France

Background: Patients with metastatic breast cancer (MBC) represent a heterogeneous group, with large differences in outcomes from individual patients. VE-cadherin, an endothelial-specific cadherin, was shown to promote tumour proliferation and angiogenesis. Soluble VE-cadherin has been recently associated to breast cancer progression. This study was designed to investigate the prognosis significance of soluble VE-cadherin in hormone-refractory MBC.

Methods: Between 2004 and 2007, 150 patients with a fully documented history of hormone-refractory MBC were included in the prospective SEMTOF study. Serum concentrations of VE-cadherin were measured at inclusion for 141 patients and 6 weeks after the beginning of chemotherapy, using a sandwich enzyme immunoassay.

Results: The presence of high levels of serum VE-cadherin was significantly correlated to a shorter progression-free (PFS) and overall survival (OS). In a multivariate analysis along with clinical and biologic prognostic parameters, high serum VE-cadherin level was an independent adverse prognostic variable for PFS (median PFS 9.7 (IC95: 8; 11.9) vs 5.8 (IC95: 4.1; 8) months $P=0.0008$ ) and OS (median OS 34 (IC95: 26.6; 47.1) vs 14.8 (IC95: 9.3; 21.4) months $P=0.0007$ ). Moreover, VE-cadherin decrease during chemotherapy was also associated with good prognosis.

Conclusions: Serum VE-cadherin levels correlate to poorer survival in patients with hormone-refractory MBC. As sVE-cadherin reflects tumour angiogenesis, this could have therapeutic implications for antiangiogenic treatment.

Vascular endothelial (VE)-cadherin, also known as cadherin 5, is an endothelial-specific cadherin localised at adherens intercellular junctions of vascular endothelial cells (Giannotta et al, 2013). VE-cadherin plays an important role in the control of vascular integrity and permeability (Dejana and Orsenigo, 2013; Bravi et al, 2014). VE-cadherin promotes tumour progression by contributing to tumour angiogenesis (Wallez et al, 2006) by interaction with VE growth factor receptor (VEGFR)-2 but also by enhancing tumour

*Correspondence: Dr T Bachelot; E-mail: Thomas.bachelot@lyon.unicancer.fr

Received 22 June 2016; revised 18 November 2016; accepted 1 December 2016; published online 5 January 2017

(c) 2017 Cancer Research UK. All rights reserved 0007-0920/17 
cell proliferation via the transforming growth factor- $\beta$ signalling pathway (Labelle et al, 2008a). Unlike most endothelial markers, VE-cadherin is not found in blood cells or in haematopoietic precursors. Cytokine-induced phosphorylation of the VE-cadherin cytoplasmic domain was reported to trigger cleavage of its extracellular domain, releasing soluble form of the proteinsoluble VE-cadherin (sVE-cadherin) (Vilgrain et al, 2013). As a consequence, sVE-cadherin may be related to neoangiogenesis and tumour burden. We have previously shown that the cleavage of VE-cadherin is dependent upon tyrosine kinase activation in response to cytokines challenge and more precisely Src kinase in response to VEGF (Wallez et al, 2007; Vilgrain et al, 2013).

Breast cancer is the most common cancer type among women and it is the leading cause of cancer mortality among women in Europe (Ferlay et al, 2013; Arnold et al, 2015). Metastatic breast cancer (MBC) is generally considered as an incurable malignancy. Although therapeutic development has improved prognostic, median survival is still limited at 24-30 months after the diagnosis of metastasis (Largillier et al, 2008). MBC is a heterogeneous disease: actual survival for individual patients varies widely, from just a few months to several years. Thus, stratification of patients into poor and good prognosis groups would lead to better personalised therapeutic approach (Ryberg et al, 2001; Chang et al, 2003; Dawood et al, 2010). Additional biological parameters are therefore required to more accurately predict outcome of $\mathrm{MBC}$ patients (Kos and Dabbs, 2016). Recent studies have shown increase levels of sVE-cadherin in MBC compared with localised breast cancer (Fry et al, 2013, 2016). The SEMTOF study was a prospective study specifically designed to identify biological prognostic factor in patients with hormone-refractory MBC. Here we focused on sVE-cadherin results and its identification as an independent prognosis factor.

\section{PATIENTS AND METHODS}

Patients. The SEMTOF study was a multicentre prospective cohort study whose aim was to collect biological samples for prognostic and predictive translational analysis in hormonerefractory $\mathrm{MBC}$ patients. All patients agreed and signed an inform consent before inclusion. This study was done following international and national regulation in vigour at that time and was approved by the institutional ethics committee on 16 March 2004. Inclusion criteria were: age $\geqslant 18$ years old, histologically proved MBC. Patients had to be eligible at inclusion to a first-line metastatic chemotherapy. Chemotherapy regimen was not predefined and was under investigator choice. All patients with HER2 positive disease received Trastuzumab associated with chemotherapy. Previous radiotherapy and hormonotherapy were allowed, patients had to be naive from metastatic chemotherapy. Progression after homone therapy was defined as relapse occurring on the first year of adjuvant hormonotherapy, or progression within first 6 months of first-line hormonotherapy for MBC. They were included between July 2004 and 2007.

After obtaining patient informed consent, serum and matched plasma samples were collected, aliquoted and stored at $-80^{\circ} \mathrm{C}$, then thawed shortly before use.

Age, Eastern Cooperative Group PS, initial tumour stage, histology, HER2/neu status, initial number of positive lymph nodes, number and sites of metastases, biological parameters at inclusion (lactate dehydrogenase, haemoglobin, neutrophils count (PNN), time since diagnosis of the initial breast cancer (diseasefree interval), prior chemotherapy or endocrine treatment (including the adjuvant setting) and the nature of the metastatic treatment were collected.
Clinical, biological and radiological evaluations were performed at inclusion, then every 6 weeks.

Serum analysis. Serum samples were kept frozen at $-80{ }^{\circ} \mathrm{C}$ and then thawed shortly before determination of sVE-cadherin level by ELISA assay. The assay was a sandwich enzyme immunoassay using a monoclonal antibody and an enzyme-linked polyclonal antibody specific for sVE-cadherin, as previously described (Vilgrain et al, 2013). Individual serum concentrations of sVEcadherin are reported in $\mu \mathrm{g} \mathrm{ml}^{-\mathrm{a}}$.

Statistical analysis. Statistical analyses were carried out using the SAS package 9.4. Patients' characteristics were described using median $\left({ }_{\min } \max \right)$ or frequency and percentage for quantitative and qualitative parameters, respectively. Overall survival was defined as the time from inclusion to death of any cause or last news for alive patients. Progression-free survival was defined as the time from inclusion to the date of first documentation of progression or the date of death or date of last follow-up. Survival data were assessed by the Kaplan-Meier method. In the absence of assumptions concerning a hypothetic cut-off, two subgroups of comparable size were used to split population depending on sVE-cadherin level. In order to maximise the power of the study, the median value of the distribution score has been used. A second threshold, $\left(0.72 \mu \mathrm{gl}^{-\mathrm{h}}\right)$ using outcome-based cut-point optimisation method defined with $\mathrm{X}$-tile software was used (Camp et al, 2004). Survival curves were established according to the initial VE-cadherin level and compared using a Log-rank test. A step by step descending selection of variables was used so as to retain factors that were independently linked to survival parameters. The variables introduced in the multivariate Cox regression model were prognostic parameters previously described as being relevant in MBC, as biological subtype or parameters previously identify as significantly related to survival in the univariate procedure (Yamamoto et al, 1998; Largillier et al, 2008; Kwast et al, 2014). Significant variable (at $10 \%$ level) in the univariate approach were introduced in the initial multivariate model. The final multivariate model was those reduced to the only variables which were statistically significant at a $5 \%$ threshold.

\section{RESULTS}

Patients' characteristics. One hundred and fifty patients were included between July 2004 and 2007. Patients' characteristics are shown in Table 1. Median age was 56.6 years (range $30-84$ ). Thirty patients $(20 \%)$ presented metastasis at initial diagnosis. Eightyseven patients had received adjuvant chemotherapy (AC, FEC, FAC and CMF). The median disease-free interval (from first diagnosis to recurrence) was 36.9 months varying from 0 to 289 months. All patients in this series had metastatic disease refractory to hormone therapy, that is, receptor-negative for both oestrogen and progesterone or progressive after hormone therapy. Twentysix patients were HER2 positive, 18 patients $(12.3 \%)$ had triple negative disease. Most patients had ductal $(n=121)$ rather than lobular $(n=12)$ histological subtype. Median soluble VE-cadherin value was not significantly different between this two groups.

Serum levels of sVE-cadherin. Initial sVE-cadherin serum level was assessed on 141 patients, with a median of $0.545 \mu \mathrm{g} \mathrm{ml}^{-\mathrm{s}}$ (range 0.01-2.62 $\mu \mathrm{g} \mathrm{ml}^{-1}$ ). A sVE-cadherin serum level at week 6 was assessed on 111 patients, with a median of $0.494 \mu \mathrm{g} \mathrm{ml}^{-1}$ (range $0.01-1.66 \mu \mathrm{g} \mathrm{ml}^{-1}$ ). A reduction of more than $10 \%$ of sVEcadherin level at week 6 was observed in 40 patients.

Correlation of sVE-cadherin to clinico-pathological characteristics. We analysed sVE-cadherin correlation to accepted prognostic factors. Higher levels of sVE-cadherin were significantly correlated to a worse PS $(P=0.048)$, a lower haemoglobin 
Table 1. Patients characteristics $(N=141)$

\begin{tabular}{|c|c|c|}
\hline & No. of patients & Percent \\
\hline \multicolumn{3}{|l|}{ Age (years) } \\
\hline $\begin{array}{l}\text { Median } \\
\text { Range }\end{array}$ & $\begin{array}{c}56.6 \\
(30-84)\end{array}$ & \\
\hline \multicolumn{3}{|l|}{ PS } \\
\hline$<2$ & 119 & $84 \%$ \\
\hline$\geqslant 2$ & 23 & $16 \%$ \\
\hline \multicolumn{3}{|c|}{ Receptor status } \\
\hline ER positive & $\begin{aligned} 102 \\
30\end{aligned}$ & $72 \%$ \\
\hline ER negative & 39 & $27 \%$ \\
\hline \multicolumn{3}{|l|}{ Her2/neu } \\
\hline Positive & 26 & $19 \%$ \\
\hline Negative & 115 & $81 \%$ \\
\hline \multicolumn{3}{|c|}{ Liver metastasis } \\
\hline Yes & 54 & $36 \%$ \\
\hline No & 96 & $64 \%$ \\
\hline \multicolumn{3}{|c|}{ Bone metastasis } \\
\hline Yes & 56 & $40 \%$ \\
\hline No & 85 & $60 \%$ \\
\hline \multicolumn{3}{|c|}{ Number of metastatic sites } \\
\hline$\leqslant 4$ & 124 & $88 \%$ \\
\hline$>4$ & 17 & $12 \%$ \\
\hline \multicolumn{3}{|c|}{ Lactate dehydrogenase } \\
\hline$\leqslant 600$ & 109 & $78 \%$ \\
\hline$>600$ & 31 & $22 \%$ \\
\hline
\end{tabular}

$(P=0.0492)$ and a lower albumin $(P=0.0391)$. On the other hand, sVE-cadherin levels were not related to lactate dehydrogenase, $\mathrm{PNN}$, disease-free interval and liver metastasis.

Prognostic value of sVE-cadherin for survival. At the time of analysis, with a median follow-up of 72.7 months, median survival of the entire patient population was 27.7 months from the date of sampling (IC95: 23.2-32.1). 125 patients (83.3\%) deceased.

High serum level of sVE-cadherin was a factor of poor prognosis whatever the sVE-cadherin threshold used (median or Xtile cut-off). Median OS was, respectively, 33.4 (IC95: 26.1-49.8) vs 21.4 (IC95: 14.8-30.5) months (log-rank test: $P=0.0063$ ) for patients whose serum level was below and above the median sVE-cadherin distribution value equals to $0.55 \mu \mathrm{g} \mathrm{ml}^{-\mathrm{d}}$ (Figure 1). This significant prognosis effect is even more noticeable when a cut-off of 0.72 is used; median OS was, respectively, 34 (IC95: 26.647.1) vs 14.8 (IC95: 9.3-21.4) months (log-rank test: $P=0.0007$ ) for patients whose serum level was below and above $0.72 \mu \mathrm{g} \mathrm{ml}^{-1}$ value (Figure 2). The death risk is twice higher in patients with sVE-cadherin level higher than $0.72 \mu \mathrm{g} \mathrm{ml}^{-1} \mathrm{HR}=2.0$ (IC95: 1.3-3.0, $P=0.0007)$.

Significant difference in median PFS was also found between patients whose sVE-cadherin level was below or above $0.72 \mu \mathrm{g} \mathrm{ml}^{-1}$ value: 9.7 (IC95: 8-11.9) vs 5.8 (IC95: 4.1-8.0) months (log-rank test: $P=0.0008$ ) (Figure 3).

Moreover, in univariate analysis, a decrease of $10 \%$ or more of sVE-cadherin between inclusion and week 6 was associated with improved OS: HR 1.65 (IC95: 1.06-2.57, log-rank test: $P=0.025$ ) (Figure 4).

Multivariate analysis. A multivariate analysis of prognostic factors using the Cox model was performed on 119 out of 150 MBC patients without missing data. The variables introduced in the multivariate Cox regression included performance status (0-1 vs 2-3); bone involvement (present vs absent), adjuvant chemotherapy, disease-free interval, haemoglobin, SBR grade

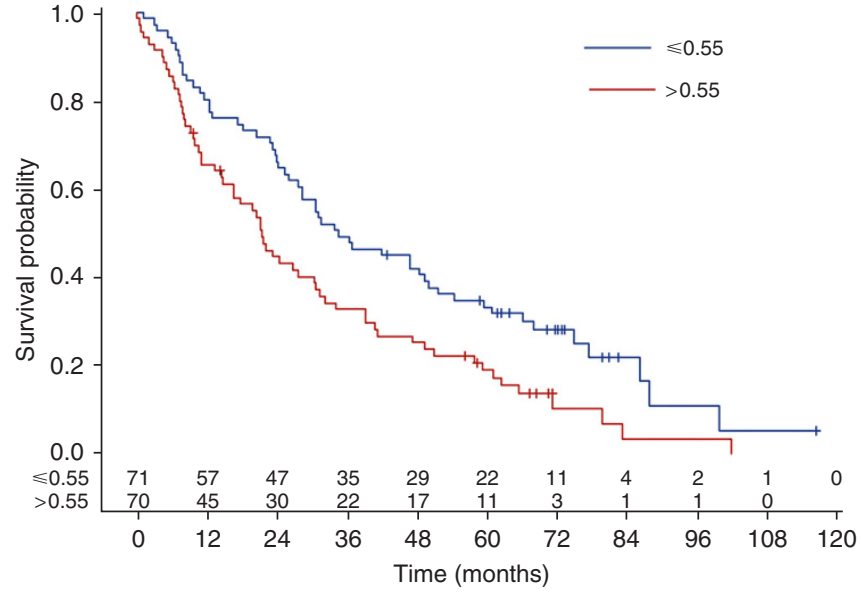

Figure 1. Impact of VE-cadherin on OS, assessed by the Kaplan-Meier method, using $0.55 \mu \mathrm{g} \mathrm{ml}^{-\mathrm{r}}$ cut-off.

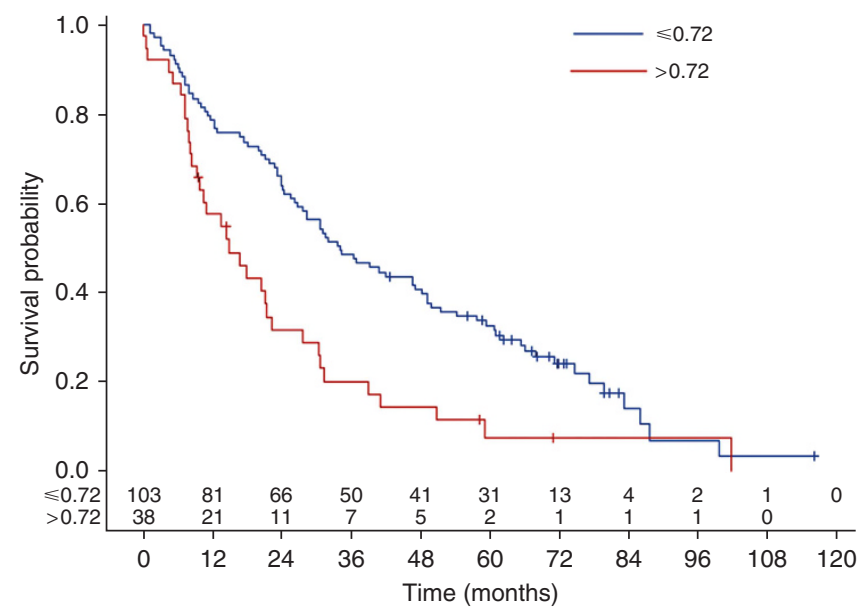

Figure 2. Impact of VE-cadherin on OS, assessed by the Kaplan-Meier method, using $0.72 \mu \mathrm{g} \mathrm{ml}^{-1}$ cut-off.

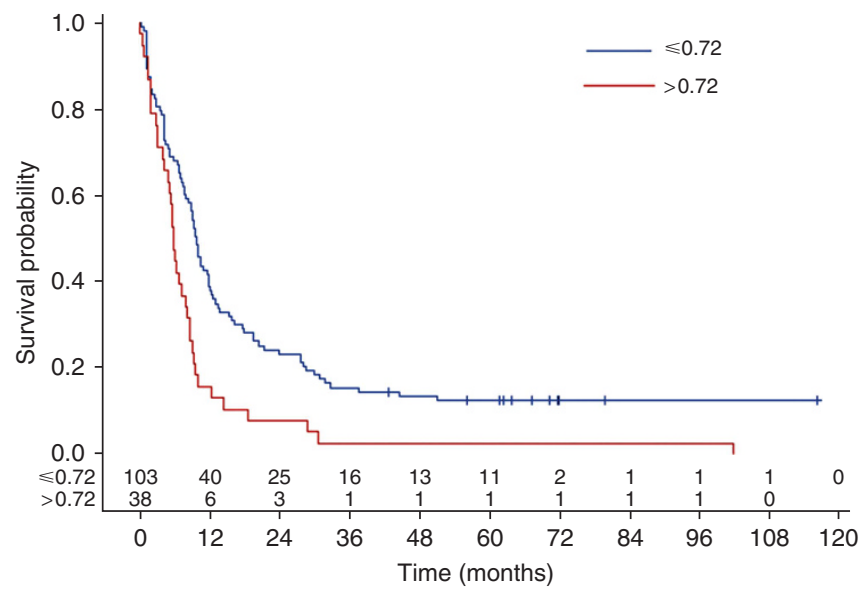

Figure 3. Impact of VE-cadherin on PFS, assessed by the KaplanMeier method, using $0.72 \mu \mathrm{g} \mathrm{ml}^{-1}$ cut-off.

(I/II $v s$ III) for OS only and sVE-cadherin level $(\leqslant 0.72 v s>0.72$ $\mathrm{ng} \mathrm{ml}^{-1}$ ). In the final model, sVE-cadherin remained an additional independent prognostic factor for OS: HR 1.672 (IC95: 1.1-2.64, $P=0.0270$ ) (Table 2) and for PFS: HR 1.612 (IC95: 1.07-2.43, $P=0.0219)$. 


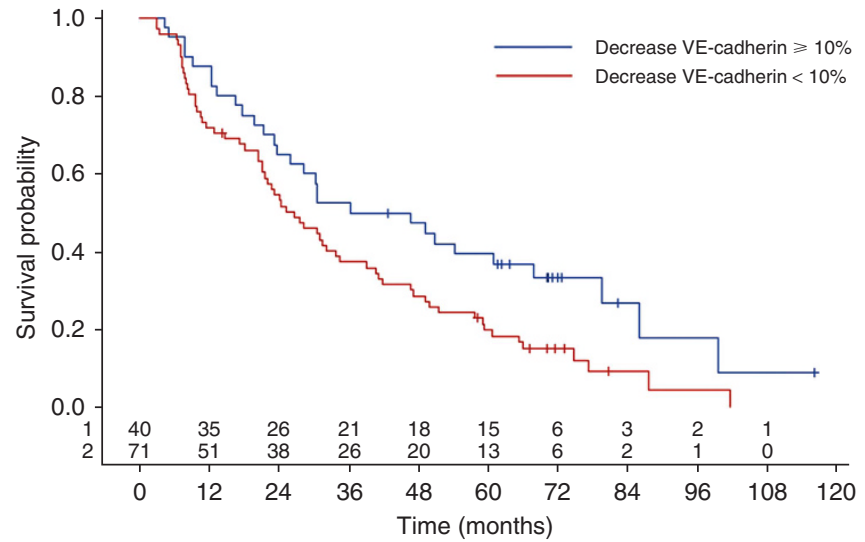

Figure 4. VE-cadherin variation during chemotherapy impact on OS, assessed by the Kaplan-Meier method.

Table 2. Results of the multivariate analysis significant factors associated overall survival and hazard ratio

\begin{tabular}{|c|c|c|c|c|}
\hline Factor & $\begin{array}{l}\text { Median } \\
\text { survival }\end{array}$ & $P$-value & HR & $\begin{array}{l}95 \% \text { confidence } \\
\text { interval of HR }\end{array}$ \\
\hline \multicolumn{5}{|l|}{ Grade } \\
\hline SBR1/2 & 39.0 & \multirow[t]{2}{*}{0.0177} & \multirow[t]{2}{*}{1.648} & \multirow[t]{2}{*}{$(1.09-2.5)$} \\
\hline SBR3 & 21.2 & & & \\
\hline \multicolumn{5}{|c|}{ Haemoglobin $\left(\mathrm{g} \mathrm{dl}^{-1}\right)$} \\
\hline$<11.5$ & 28.4 & \multirow[t]{2}{*}{0.0066} & \multirow[t]{2}{*}{2.092} & \multirow[t]{2}{*}{$(1.22-3.56)$} \\
\hline$\geqslant 11.5$ & 10.8 & & & \\
\hline \multicolumn{5}{|c|}{ Bone metastasis } \\
\hline Yes & 41.1 & \multirow[t]{2}{*}{0.0009} & \multirow[t]{2}{*}{2.096} & \multirow[t]{2}{*}{$(1.35-3.25)$} \\
\hline No & 19.0 & & & \\
\hline \multicolumn{5}{|c|}{ VE-cadherin $\left(\mu \mathrm{g} \mathrm{ml}^{-1}\right)$} \\
\hline$\leqslant 0.72$ & 28.4 & \multirow[t]{2}{*}{0.027} & \multirow[t]{2}{*}{1.672} & \multirow[t]{2}{*}{$(1.1-2.64)$} \\
\hline$>0.72$ & 16.6 & & & \\
\hline
\end{tabular}

\section{DISCUSSION}

Metastatic breast cancer is a heterogeneous disease with variable biological, clinical behaviours and for any specific patient, clinical evolution is difficult to predict. New prognostic and predictive factors are therefore needed to help clinicians choosing customised therapeutic strategy. Blood-based biomarkers have advantages over tissue markers as they are easily accessible and can also be routinely monitored.

Modification of VE-cadherin (or cadherin 5) expression has been observed in several cancer types, including breast cancer. In preclinical models, aberrant expression of VE-cadherin promotes tumour progression via the TGF- $\beta$ pathway that leads to cell proliferation and to epithelial-mesenchymal transition, which is a phenotypic alteration associated with metastatic dissemination and progression (Labelle et al, 2008b; Fry et al, 2016).

VE-cadherin cytoplamic domain contain several tyrosine residues that are targeted by tyrosine kinases upon cytokines challenge. We have previously shown that VEGF-induced VE-cadherin tyrosine phosphorylation at site Y685 upon Src kinase activation (Wallez et al, 2007). In addition, we have demonstrated that VEGF-induced VE-cadherin tyrosine phosphorylation preceded N-terminal ectodomain shedding of the protein (Vilgrain et al, 2013). The mechanism of VE-cadherin cleavage is not completely understood. One hypothesis is that the covalent tyrosine phosphorylation in the cytoplasmic domain might induce a transconformation of the protein that could be more susceptible to proteolysis.

In this study, we have evaluated the clinical significance of soluble VE-cadherin levels in 141 hormone-refractory $\mathrm{MBC}$ patients. We demonstrate that sVE-cadherin is an independent prognostic factor in MBC for both PFS and OS. As sVE-cadherin had never been evaluated in this setting, we explored two thresholds based on statistical prediction. Elevated sVE-cadherin level $(>0.72)$ was significantly associated with worse OS and PFS. Results of the multivariate analysis show that sVE-cadherin levels remain independent variables for survival when assessed with confirmed prognostic markers. Furthermore, a decrease of $\geqslant 10 \%$ of sVE-cadherin level during chemotherapy was significantly associated with good prognosis.

Previous studies from Fry et al identified sVE-cadherin as a biomarker that distinguished patients with recurrent breast cancer from those with complete remission (Fry et al, 2013, 2016). These studies indicate that sVE-cadherin might be use as a biomarker to monitor patients after primary breast cancer treatment. This biomarker appears to be more sensitive than CA15.3. Those results indicate that measurements of sVE-cadherin could be integrated in the surveillance of patients with localised breast cancer, to early detect metastatic recurrence. Our study evaluated sVEcadherin prognostic value on patients with established MBC. We demonstrated that sVE-cadherin value and evolution can discriminate patients with $\mathrm{MBC}$ into poor and good prognosis groups, which might help physicians to better personalised therapeutic approach. Due to different clinical situation and different ELISA technics, sVE-cadherin levels in our study cannot be directly compared with Fry et al values. Nevertheless, taken together those results highlight the informative value of sVE-cadherin in MBC.

In the absence of references concerning sVE-cadherin value in $\mathrm{MBC}$, we chose in a first intention a cut-off based on median value. Cut-off of 0.72 was determined using X-tile software. It provides a global assessment of every possible way of dividing a population into low-high level marker expression. Additional analyses of sensitivity shown that the results did not vary when the cutoff changes. However, these cut-off can be discussed as they have no biological rational even if, they are twice associated with significantly statistical differences. Furthermore, we wanted to identify if variation of VE-cadherin level in the first weeks of treatment could be predictive of treatment response. We chosed $10 \%$ cut-off in order to isolate patients with largest decreases in VE-cadherin at week 6 compared with baseline while having a sufficient sample size in this subgroup to keep enough statistical power to show a predictive effect of this evolution.

One other limit of our study is the heterogeneity of our cohort: we included patients with hormone receptor negative or positive disease and HER2 positive and negative disease. Because of our limited effective, we could not study either of the subtypes separately. Twenty-six patients had HER2-positive disease and they all received trastuzumab associated with metastatic first-line chemotherapy. However, soluble VE-cadherin median value was not statistically different in the different biological subtypes

Phase III trials in MBC reported PFS benefit of the adjunction of bevacizumab to taxanes but failed to demonstrate OS benefit (Miles et al, 2010; Brufsky et al, 2011; Robert et al, 2011). Part of the problem with bevacizumab development is the lack of predictive biomarker which could select for bevacizumab sensibility (Trédan et al, 2015). VEGF and VE-cadherin have been identified as mediator of tumour angiogenesis, a process required for invasion and metastasis of solid tumours (Weidner et al, 1991; Le Guelte et al, 2011). Although the role of VEGF in tumour progression has been largely demonstrated, studies failed to 
identify soluble VEGF level as a predictive biomarker (Bachelot et al, 2003; Reeves et al, 2009). Vilgrain et al demonstrated in patients with glioblastoma that VEGF can induce VE-cadherin cleavage and consequently induce sVE-cadherin release. As it is a specific component of the endothelial cells and because it has not been reported to be trapped by extracellular matrix, soluble VEcadherin in blood might reflect VEGF activity at tumour site (Vilgrain et al, 2013).Thus, sVE-cadherin might be a predictive biomarker of antiangiogenic treatment efficiency. This hypothesis is currently being tested in the prospective COMET study, which investigate various biological parameters that could be related to clinical benefit of bevacizumab in MBC.

In conclusion, we show that soluble VE-cadherin level is independently correlated with survival in hormone-refractory MBC. If confirmed, these results could help oncologists in identifying patients with a poor prognosis. Furthermore, as sVE-cadherin reflects active angiogenesis at tumour site, its evaluation might help to predict efficacy of antiangiogenic molecules.

\section{ACKNOWLEDGEMENTS}

This work was supported by the French National Institute of Health and Medical Research INSERM (UMRS 1036), the French Atomic Energy and Alternative Energies Commission (CEA), Department for Fundamental Research (DRF)/Institute of Biosciences and Biotechnologies of Grenoble: (BIG)/Invasion Mechanisms in Angiogenesis and Cancer (IMAC team)/Grenoble University Hospital, Institut National du Cancer (INCa) French Association against cancer (ARC Foundation), Fédération Nationale des Centres de Lutte contre le Cancer (GEFLUC Grenoble-Dauphiné -Savoie), Comité de la Loire et de la Saone et Loire de Ligue contre le Cancer. HP and AK-M were recipients of a grant from Association pour la Recherche contre le Cancer (ARC Foundation). We are indebted to Melanie Arboleas for technical assistance. The funders had no role in the study design, data collection and analysis, decision to publish or preparation of the manuscript.

\section{CONFLICT OF INTEREST}

The authors declare no conflict of interest.

\section{REFERENCES}

Arnold M, Karim-Kos HE, Coebergh JW, Byrnes G, Antilla A, Ferlay J, Renehan AG, Forman D, Soerjomataram I (2015) Recent trends in incidence of five common cancers in 26 European countries since 1988: analysis of the European Cancer Observatory. Eur J Cancer 51: 1164-1187.

Bachelot T, Ray-Coquard I, Menetrier-Caux C, Rastkha M, Duc A, Blay J-Y (2003) Prognostic value of serum levels of interleukin 6 and of serum and plasma levels of vascular endothelial growth factor in hormone-refractory metastatic breast cancer patients. Br J Cancer 88: 1721-1726.

Bravi L, Dejana E, Lampugnani MG (2014) VE-cadherin at a glance. Cell Tissue Res 355: 515-522.

Brufsky AM, Hurvitz S, Perez E, Swamy R, Valero V, O’Neill V, Rugo HS (2011) RIBBON-2: a randomized, double-blind, placebo-controlled, phase III trial evaluating the efficacy and safety of bevacizumab in combination with chemotherapy for second-line treatment of human epidermal growth factor receptor 2-negative metastatic breast cancer. J Clin Oncol 29: 4286-4293.

Camp RL, Dolled-Filhart M, Rimm DL (2004) X-tile: a new bio-informatics tool for biomarker assessment and outcome-based cut-point optimization. Clin Cancer Res 10: 7252-7259.
Chang J, Clark GM, Allred DC, Mohsin S, Chamness G, Elledge RM (2003) Survival of patients with metastatic breast carcinoma: importance of prognostic markers of the primary tumor. Cancer 97: 545-553.

Dawood S, Broglio K, Ensor J, Hortobagyi GN, Giordano SH (2010) Survival differences among women with de novo stage IV and relapsed breast cancer. Ann Oncol 21: 2169-2174.

Dejana E, Orsenigo F (2013) Endothelial adherens junctions at a glance. J Cell Sci 126: 2545-2549.

Ferlay J, Steliarova-Foucher E, Lortet-Tieulent J, Rosso S, Coebergh JWW, Comber H, Forman D, Bray F (2013) Cancer incidence and mortality patterns in Europe: estimates for 40 countries in 2012. Eur J Cancer 49: 1374-1403.

Fry SA, Robertson CE, Swann R, Dwek MV (2016) Cadherin-5: a biomarker for metastatic breast cancer with optimum efficacy in oestrogen receptor-positive breast cancers with vascular invasion. Br J Cancer 114: 1019-1026.

Fry SA, Sinclair J, Timms JF, Leathem AJ, Dwek MV (2013) A targeted glycoproteomic approach identifies cadherin-5 as a novel biomarker of metastatic breast cancer. Cancer Lett 328: 335-344.

Giannotta M, Trani M, Dejana E (2013) VE-cadherin and endothelial adherens junctions: active guardians of vascular integrity. Dev Cell 26: 441-454.

Kos Z, Dabbs DJ (2016) Biomarker assessment and molecular testing for prognostication in breast cancer. Histopathology 68: 70-85.

Kwast ABG, Voogd AC, Menke-Pluijmers MBE, Linn SC, Sonke GS, Kiemeney LA, Siesling S (2014) Prognostic factors for survival in metastatic breast cancer by hormone receptor status. Breast Cancer Res Treat 145: 503-511.

Labelle M, Schnittler HJ, Aust DE, Friedrich K, Baretton G, Vestweber D, Breier G (2008a) Vascular endothelial cadherin promotes breast cancer progression via transforming growth factor beta signaling. Cancer Res 68: 1388-1397.

Labelle M, Schnittler HJ, Aust DE, Friedrich K, Baretton G, Vestweber D, Breier G (2008b) Vascular endothelial cadherin promotes breast cancer progression via transforming growth factor signaling. Cancer Res 68: $1388-1397$.

Largillier R, Ferrero J-M, Doyen J, Barriere J, Namer M, Mari V, Courdi A, Hannoun-Levi JM, Ettore F, Birtwisle-Peyrottes I, Balu-Maestro C, Marcy PY, Raoust I, Lallement M, Chamorey E (2008) Prognostic factors in 1038 women with metastatic breast cancer. Ann Oncol 19: 2012-2019.

Le Guelte A, Dwyer J, Gavard J (2011) Jumping the barrier: VE-cadherin, VEGF and other angiogenic modifiers in cancer. Biol Cell 103: 593-605.

Miles DW, Chan A, Dirix LY, Cortés J, Pivot X, Tomczak P, Delozier T, Sohn JH, Provencher L, Puglisi F, Harbeck N, Steger GG, Schneeweiss A, Wardley AM, Chlistalla A, Romieu G (2010) Phase III study of bevacizumab plus docetaxel compared with placebo plus docetaxel for the first-line treatment of human epidermal growth factor receptor 2-negative metastatic breast cancer. J Clin Oncol 28: 3239-3247.

Reeves KW, Ness RB, Stone RA, Weissfeld JL, Vogel VG, Powers RW, Modugno F, Cauley JA (2009) Vascular endothelial growth factor and breast cancer risk. Cancer Causes Control 20: 375-386.

Robert NJ, Diéras V, Glaspy J, Brufsky AM, Bondarenko I, Lipatov ON, Perez EA, Yardley DA, Chan SYT, Zhou X, Phan S-C, O'Shaughnessy J (2011) RIBBON-1: randomized, double-blind, placebo-controlled, phase III trial of chemotherapy with or without bevacizumab for first-line treatment of human epidermal growth factor receptor 2-negative, locally recurrent or metastatic breast cancer. J Clin Oncol 29: $1252-1260$.

Ryberg M, Nielsen D, Osterlind K, Skovsgaard T, Dombernowsky P (2001) Prognostic factors and long-term survival in 585 patients with metastatic breast cancer treated with epirubicin-based chemotherapy. Ann Oncol 12: $81-87$.

Trédan O, Lacroix-Triki M, Guiu S, Mouret-Reynier M-A, Barrière J, Bidard F-C, Braccini A-L, Mir O, Villanueva C, Barthélémy P (2015) Angiogenesis and tumor microenvironment: bevacizumab in the breast cancer model. Target Oncol 10: 189-198.

Vilgrain I, Sidibé A, Polena H, Cand F, Mannic T, Arboleas M, Boccard S, Baudet A, Gulino-Debrac D, Bouillet L, Quesada J-L, Mendoza C, Lebas J-F, Pelletier L, Berger F (2013) Evidence for post-translational processing of vascular endothelial (VE)-cadherin in brain tumors: towards a candidate biomarker. PLoS One 8: e80056.

Wallez Y, Vilgrain I, Huber P (2006) Angiogenesis: the VE-cadherin switch. Trends Cardiovasc Med 16: 55-59. 
Wallez Y, Cand F, Cruzalegui F, Wernstedt C, Souchelnytskyi S, Vilgrain I, Huber P (2007) Src kinase phosphorylates vascular endothelial-cadherin in response to vascular endothelial growth factor: identification of tyrosine 685 as the unique target site. Oncogene 26: 1067-1077.

Weidner N, Semple JP, Welch WR, Folkman J (1991) Tumor angiogenesis and metastasis-correlation in invasive breast carcinoma. $N$ Engl J Med 324: $1-8$.

Yamamoto N, Watanabe T, Katsumata N, Omuro Y, Ando M, Fukuda H, Takue Y, Narabayashi M, Adachi I, Takashima S (1998) Construction and validation of a practical prognostic index for patients with metastatic breast cancer. J Clin Oncol 16: 2401-2408.

This work is published under the standard license to publish agreement. After 12 months the work will become freely available and the license terms will switch to a Creative Commons AttributionNonCommercial-Share Alike 4.0 Unported License. 